\title{
Recognization and Analysis of Key News Event for Chinese Stock Market Based on Change Point Analysis and Event Study
}

\author{
Li Qingyang ${ }^{1, a}$, Wang Yue ${ }^{2, b,{ }^{*}}$ and Wu Jiachen ${ }^{3, c}$ \\ ${ }^{1}$ School of Information, Central University of Finance and Economics, Baishan Town, Changping, \\ Beijing, China \\ 2 School of Information, Central University of Finance and Economics, Baishan Town, Changping, \\ Beijing, China \\ ${ }^{3}$ School of Information, Central University of Finance and Economics, Baishan Town, Changping, \\ Beijing, China \\ a1085191646@qq.com, b5 53752466@qq.com, c 525588910@qq.com \\ ${ }^{*}$ Corresponding author
}

Keywords: Changepoint Analysis, Event Study, CSI 300 Index.

\begin{abstract}
We used the automatic keyword extraction algorithm to identify the key events of the obtained news reports and get 45 key events. We further defined the method of calculating the relevance of events, applying the lexical field theory, and build an event correlation network.

Then we used change point analysis to extract the stage change of the market index. The common parts of the various algorithm frameworks and research objects are regarded as reliable change points, and the time of a change point is used to screen the 45 key events, and the seven majors in 2017 are obtained.

Finally, we use event research to analyze the impact of these seven key events on the market's yield and the differences between these effects. optional.
\end{abstract}

\section{基于变点分析和事件研究法的A股关键新闻事件识别与分析 \\ 李清扬 ${ }^{1, a}$, 王悦 $2, \mathrm{~b},{ }^{*}$, 吴佳辰 ${ }^{3, c}$ \\ 1中央财经大学信息学院, 百善镇, 昌平, 北京, 中国 \\ 2中央财经大学信息学院, 百善镇, 昌平, 北京, 中国 \\ 3中央财经大学信息学院, 百善镇, 昌平, 北京, 中国 \\ a1085191646@qq.com, b53752466@qq.com, c525588910@qq.com \\ *通讯作者}

关键词：变点分析；事件研究法；沪深300

中文摘要. 我们应用词汇场理论，定义了计算事件相关度的方法，构建起事件关联网络。随 后我们用变点分析提取大盘指数及其收益率的阶段性变点，以多种算法框架和研究对象的变 点分析结果的公共部分作为可靠变点, 用变点的时间点来䇻选事件, 得到 2017 年的七个关键 事件。最终我们用事件研究法分析这七个关键事件对大盘收益率的影响，以及这些影响之间 的差异。 


\section{1. 引言与文献综述}

股市的变动往往受到很多因素的影响，从上世纪开始，互联网财经新闻对股市的影响开 始被学者研究。但毕竟从基本面分析的角度来看, 公司官网发布的信息, 其效用应该高于财 经新闻网站提供的信息。所以我们选择用变点分析法进一步篮选新闻事件，以确保它们对股 市影响的显著性。

在整个研究过程中, 我们借鉴了徐有情[3]设置多种长度和位置的事件窗, 以确定事件具 体影响范围的方法, 并用R语言实现并优化了康宏的算法[2]。在时间序列建模预测的过程中 我们用R语言实现了杨帷的模型确立与检验方法[4]。在结果的讨论中我们借鉴了陈金短期冲 击和累积效应的理论分析框架[1]。

\section{2. 关键事件的篮选}

我们首先对爬取到的 2017 年的 30000 余篇财经新闻标题和文本的进行关键词识别, 并通过 关键词聚类实现事件的识别, 共发现 45 个事件。

\section{1 变点分析方法概述}

变点分析在金融领域属于一种比较新颖的统计学方法，常用于对金融时间序列进行阶段 划分等技术分析。两个核心方法块构成它的基本单元：一是对CUMSUM进行累计求和并进行 Bootstrap; 二是用MSE最小原则选定变点的准确位置。

为了探寻多个变点的位置, 两个基本方法模块需要进行进一步组织。组织形式有简单二 分法（Binary Segment）与后向笁除结合迭代优化、邻近分割法（Segment Neighborhood）以 及精确修剪的线性时间模型（PELT）三种。

变点分析根据MSE最小原则的作用对象又可以分为mean、var以及meanvar三种处理方法。 其中mean方法单纯考虑单期收益率大小的变动; var方法仅基于单期收益率的波动性; meanvar 将二者加权考量。

\section{2 实证与结果}

我们采用了 2017 全年的沪深 300 指数, 尝试了框架和对象的组合, 笁除了过于密集的分段 结果, 得到的结果如图1所示:
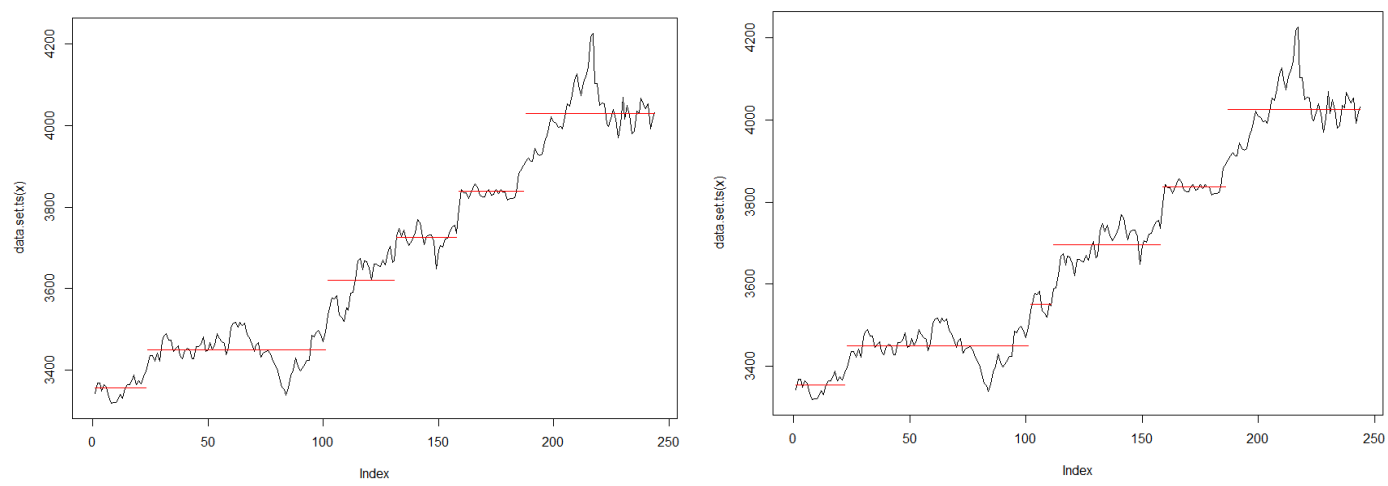

图1 BinSeg框架下对指数本身分别使用mean()方法和meanvar()方法的结果

我们将大盘指数的变点日期取交集, 并加入收益率的一个变点, 找到较为可靠的 7 个公共 变点, 并将这些时间点映射到我们的事件集（共有45个新闻事件）中, 结果如表所示: 
表1 可靠变点及其相应即期事件

\begin{tabular}{|l|l|l|l|l|}
\hline 基于均值的BinSeg & $\begin{array}{l}\text { 基于均值和方差的 } \\
\text { Binseg }\end{array}$ & $\begin{array}{l}\text { 基于均值和方差的 } \\
\text { PELT }\end{array}$ & 发生日 & 事件内容 \\
\hline $2017-02-09$ & $2017-02-08$ & $2017-02-09$ & $2.11-2.20$ & 证监会规范再融资 \\
\hline $2017-06-06$ & $2017-06-06$ & $2017-06-06$ & 6.1 & 减持新规发布 \\
\hline & $2017-06-20$ & $2017-06-22$ & 6.20 & A股被纳入MSCI \\
\hline $2017-07-18$ & $2017-07-18$ & 7.18 & 创业板暴跌5\% \\
\hline $2017-08-24$ & $2017-08-24$ & $2017-08-25$ & $8.20-8.24$ & 联通混改方案落地 \\
\hline $2017-10-11$ & $2017-10-10$ & & 10.10 & 茅台带领白酒行业创新高 \\
\hline & 收益率变点: $2017-11-20$ & & 资管新规发布 \\
\hline
\end{tabular}

\section{3. 关键事件影响分析}

\section{1 确定事件窗和估计窗}

考虑到重大事件的影响周期较长, 我们将事件窗长度控制在20天左右; 另外考虑到估计 精确度，将估计窗定为 2 个月，具体起止日期如表2所示：

表2 事件的事件窗和估计窗

\begin{tabular}{|c|c|c|c|}
\hline 关键事件 & 发生日 & 事件窗 & 估计窗 \\
\hline 证监会规范再融资 & $2.11-2.20$ & $2.5-2.28$ & $1.4-2.4$ \\
\hline 减持新规发布 & 6.1 & $5.24-6.7$ & $3.23-5.23$ \\
\hline A股被纳入MSCI & 6.20 & $6.13-6.26$ & $4.12-6.12$ \\
\hline 创业板暴跌5\% & 7.18 & $7.12-7.25$ & $5.11-7.11$ \\
\hline 联通混改方案落地 & $8.20-8.24$ & $8.17-9.2$ & $6.16-8.16$ \\
\hline 茅台带领白酒行业创新高 & 10.10 & $9.27-10.17$ & $7.26-9.26$ \\
\hline 资管新规发布 & 11.17 & $11.12-11.24$ & $9.11-11.11$ \\
\hline
\end{tabular}

\subsection{CAR相关统计量的计算和分析}

我们选用了ARIMA (4, 0，12) 模型, 以估计窗内的收益率时间序列预测事件窗内的, 记为正常收益率 $\left(C R_{t}\right)$ 。然后通过实际收益率 $\left(R_{t}\right)$ 计算超额收益率 $\left(A R_{i}\right)$ 、累积超额收 益率 $\left(C A R_{i}\right)$ 、平均累积超额收益率 $(C A R)$ 。

$$
\begin{array}{r}
A R_{t}=R_{t}-C R_{t} \\
C A R_{i}=\sum_{t=1}^{i} A R_{t} \\
A C A R_{i}=\frac{1}{N} C A R_{i}
\end{array}
$$

对 7 个事件CAR显著不为零进行 $\mathrm{t}$ 检验, 均通过。故可以对事件窗口中的 $C A R_{i}$ 进行进一步 分析。量化的统计表如表3 所示:

表3 事件的事件窗和估计窗

\begin{tabular}{|l|l|l|l|}
\hline 事件 & CAR均值 & CAR极差 & CAR标准差 \\
\hline 证监会规范再融资 & 0.022 & 0.030 & 0.008 \\
\hline 减持新规发布 & 0.005 & 0.023 & 0.008 \\
\hline A股被纳入MSCI & -0.001 & 0.033 & 0.010 \\
\hline 创业板暴跌5\% & -0.008 & 0.021 & 0.007 \\
\hline 联通混改方案落地 & 0.005 & 0.021 & 0.006 \\
\hline 茅台带领白酒行业创新高 & 0.009 & 0.021 & 0.009 \\
\hline 资管新规发布 & -0.004 & 0.033 & 0.012 \\
\hline
\end{tabular}


证监会规范再融资, A股被纳入MSCI, 资管新规发布三个事件事件窗内累计超额收益序 列的极差和标准差较大, 说明对股市的影响存在明显波动。

$\mathrm{A}$ 股被纳入MSCI，创业板暴跌 $5 \%$ ，资管新规发布三个事件事件窗内累计超额收益序列均 值为负，说明事件短期影响为利空。

\section{4. 结论和展望}

本文仅对单个事件的影响进行量化分析和比对，得到的分析结果与我们对金融信息影响 的预期是一致的。

希望今后能够实现的改进有:

1. 结合市场有效性的波动，分析新闻信息影响的显著性;

2. 整合相关事件进行主成分分析;

3. 除了对大盘指数进行研究, 也对各个行业指数进行分析。

\section{References}

[1] Chen Xin, Testing the Short-term Shock and Cumulative Effect of China's Interest Rate Policy on Stock Market [D], Fudan University, 2013.

[2] Kang Hong, Research and Design of Event Study Algorithms [D], Hebei University of Technology, 2005.

[3] Yang Jidong, Does the media influence investor behavior? — A Consideration Based on Documentation, Financial Research, vol.11, 2017.

[4] Yang Wei, Empirical Analysis of Shanghai Composite Index Based on ARIMA-GARCH Model [D], Xiangtan University, 2017. 